\title{
The thumb: Guidelines for a robotic design
}

\author{
Maxime Chalon, Markus Grebenstein, Thomas Wimböck, Gerd Hirzinger \\ Institute of Robotics and Mechatronics, German Aerospace Center (DLR), Wessling, Germany \\ E-mails: \{Maxime.Chalon,Markus.Grebenstein,Thomas.Wimboeck\}@dlr.de
}

\begin{abstract}
The impressive manipulation capabilities of the human hand are undoubtedly related to the thumb opposition. Such a versatility is highly desirable in the context of humanoid robots, in particular when performing object manipulation. Biomechanical data, surgery procedures and rehabilitation surveys represent an excellent base from which a robotic design can be inferred. This knowledge must be understood to identify the properties required for manipulation skills, and especially, to obtain a holistic view of the thumb functionality. Several designs have been realized, that concentrated on biomimetism or on classical mechanism designs. Therefore, it is currently difficult for designers to obtain a clear overview of the properties required for a functional robot thumb.

In the present case, a robotic hand with size, forces, velocity and shape comparable to the human ones, is envisioned. Unlike most of robotic designs - where the fingers are modular and the thumb is simply a finger placed in opposition - the thumb benefits from an intensive functional analysis. This paper gathers anatomy, surgery and rehabilitation data and identifies the properties required for human like manipulation. Based on this synergy, guidelines are presented that are fused and applied to the hand design of the Integrated Hand arm project of DLR.
\end{abstract}

\section{INTRODUCTION}

Despite the growing interest in robotic hands, little material proposes clear guidelines for the design of an anthropomorphic hand and especially the thumb. Although its central role in the human performance has been acknowledged for decades, roboticists only focused sparsely on the special role of the thumb. On the contrary, this importance is well covered by the medical knowledge. For John Napier, the hand without a thumb is at worst nothing but an animated spatula and at best a pair of forceps whose points don't meet properly. Thus, it is no surprise that some insurances give to the thumb loss twice the value of other fingers [1].

The difficulty of building and maintaining small integrated systems certainly explains that the robotic hands are often using a modular design for the fingers [2]. These designs are adapted, but not designed specifically for the thumb. The lack of synthesis makes it difficult for the designer to obtain an idea of the desirable features. The data concerning forces, stiffness or range of motion exist but are spread across many documents. Moreover, the precise modeling of the biological system is not useful for the designer, since it has design constraints (actuation, bearings, surfaces wear, etc...) that impose choices different from the one living material has (muscles fibers, cartilages, self healing surfaces). The designer requires an abstraction of the functionality to create effective solutions.

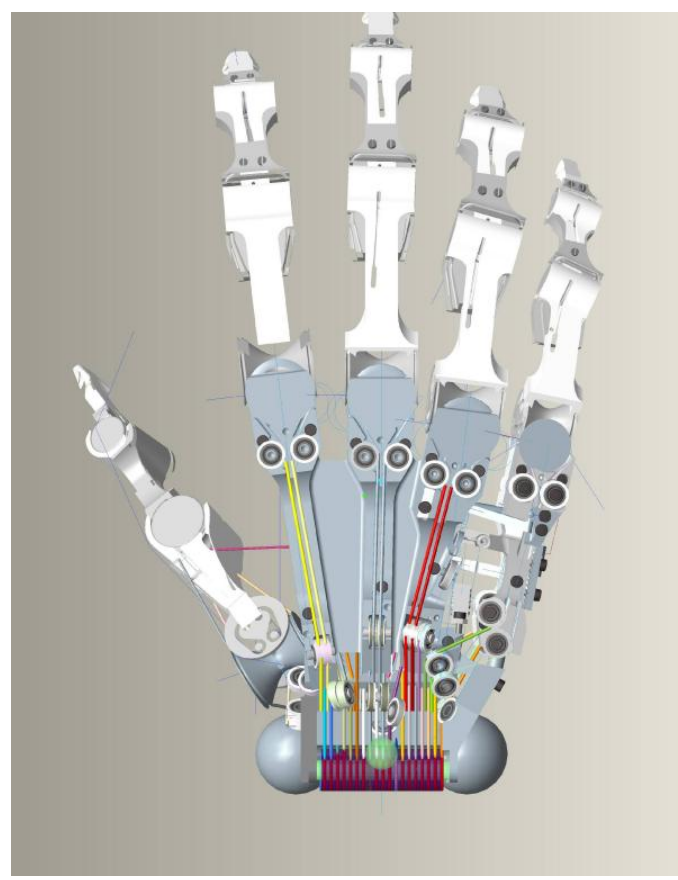

Fig. 1. CAD model of the hand skeleton (top view)

The modeling of hands has been largely discussed from a biomedical point of view, for example in the context of brain machine interfaces (BMI, cf. [3]). Several models of the human hand have been built at different granularity levels, but they focused on the identification of a model (e.g. kinematic model [4], [5], tendon forces distribution [6]) and not the functional aspects. Previous approaches followed the objective of creating an anatomically correct system (eg. ACT hand [7]). They mimicked the configuration of the bones and of the tendons to match very closely the one of humans. Work has also been done on multifingered manipulation [8] but with less concern on the degree of anthropomorphism. In this paper, the approach is to understand the key properties of the thumb functionality in order to design a high performance hand. The hand is to be of comparable size, dexterity and strength as humans (which is a common limitation of current hand designs), while keeping a strong biomimetic inspiration (other examples of such designs are : the Twendy-one hand [9], the Shadow hand [10], the Gifu hand [11]). This paper contributes to the design of the new integrated hand arm system of the DLR (cf. figure $1,[12])$. 


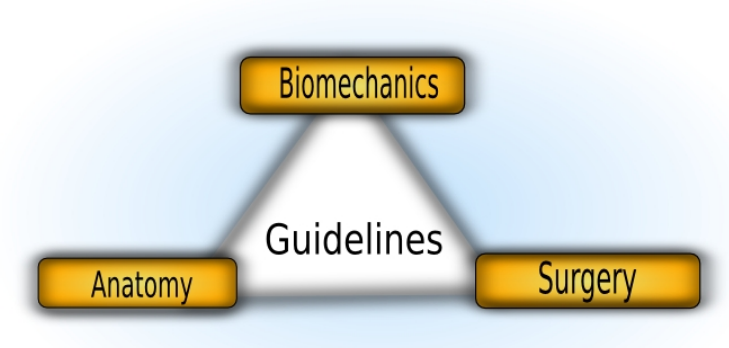

Fig. 2. Synergy of the medical knowledge, biomedical data and anatomy to generate guidelines

In this paper major anatomy elements, bones, tendons, joints and muscles, are presented to the designer. Their relevant aspects for the functionality of the thumb are extracted from surgery acts on the thumb reconstruction. The functional importance of the joints mobility is analyzed through the results of rehabilitation literature. The knowledge contained in the medical domain is synthesized and presented into a "engineer readable" form. Figure 2 represents how the medical knowledge, biomechanical data and anatomy are fused to generate the design guidelines. Since the objective of the designer is to obtain a functional thumb, and not a mechanical copy of a biological system (which is far from reach of current technologies), we derive design rules and idioms rather than absolute values. Indeed, observing the great diversity of thumb sizes and shapes, it can be hypothesized that the exact values of the mechanical properties of the thumb are not the key of a functional design.

In the first section, inputs from anatomy are presented. The structure of the thumb is presented and biomedical data is reported. The second section discusses several thumb surgery procedures and highlights the functional role of the joints. Section three presents three functional evaluation tests and analyzes the results in order to asset the relative importance of the joints. At the end of each section, the main ideas are listed. Finally, the guidelines are gathered and redundancies or discrepancies are emphasized. The guidelines have been applied to the hand of the new integrated hand arm system of the DLR (cf. figure 1, [12]), and the resulting design is briefly presented in section four.

\section{Simplified ANATOMY}

In the context of robotic hand design, the most relevant groups are the bones, the muscles and the tendons for the actuation apparatus. The skin and the muscle spindles for the sensing apparatus. This paper concentrates on actuation apparatus and therefore the skin properties are not considered. It must be noted however, that human hands include many of other biological subsystems like the cartilages, the nerves and the blood vessels.

Bones: The thumb is composed of 2 phalanxes (unlike the other fingers that have 3 ) and a metacarpal. Figure 3

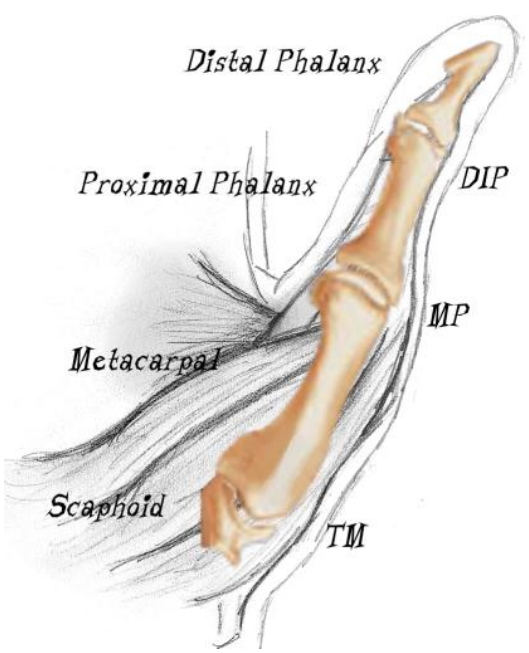

Fig. 3. Joints and bones of the hand

presents the thumb bones and joint names. The base of the thumb is the Trapezoid-Metacarpal joint (TM) whereas the most distal part is the Distal-Inter-Phalangeal joint (DIP). The middle joint is the Proximal Inter Phalangal joint (PIP). The central joint is called Metacarpal-Proximal (MP) joint.

Below the base of the thumb are several little bones, the carpal bones (trapezoid and scaphoid), that are not of major importance for the thumbs abilities due to their small range of motion.

The length of the bones have a broad distribution (between men and women, children and adults) although all of them perform well during manipulation task. Consequently, the absolute values are not relevant but rather the ratios between the joint to joint distances. Table I reports the ratios found in [13] (the thumb values were not included in the survey but similar pattern most likely apply). It appears that the ratios are very similar among individuals. Certainly, a functional robotic hand would benefit from following similar rules for the ratios of the length of the links.

TABLE I

Distance Ratios Between the PhalanXes Lengths (WITH A 95\% CONFIDENCE INTERVAL, CI)

\begin{tabular}{l|lll} 
Digit & $\begin{array}{l}\text { MP-PIP/PIP- } \\
\text { Tip } \\
\text { Ratio (95\% CI) }\end{array}$ & $\begin{array}{l}\text { MP-PIP/PIP-DIP } \\
\text { Ratio (95\% CI) }\end{array}$ & Ratio (95\% CI) \\
\hline Index & $1.02(0.006)$ & $1.86(0.018)$ & $1.24(0.018)$ \\
Middle & $0.99(0.004)$ & $1.72(0.013)$ & $1.36(0.016)$ \\
Ring & $0.95(0.007)$ & $1.70(0.016)$ & $1.29(0.016)$ \\
Little & $0.98(0.007)$ & $1.91(0.022)$ & $1.06(0.022)$
\end{tabular}

Muscles and tendons: Eight muscles are used to control the thumb motion. Part of them are directly located in the palm and provide an increased lever arm (thus, greater forces and sensitivity). They are called thenarian muscles (the little finger has similar muscles called hypothenarian muscles). 
The others are extrinsic muscles situated in the forearm and linked to the bones via tendons. The tendon insertions ${ }^{1}$ are not simple points and, for example, the TM extensor is attached to the bone and an intrinsic muscle at the same time. Consequently, the determination of the tendon contribution is position dependent and non-linear [6]. The following figures present an overview of the muscles and tendons of the thumb (more details are available in anatomy books [14]).

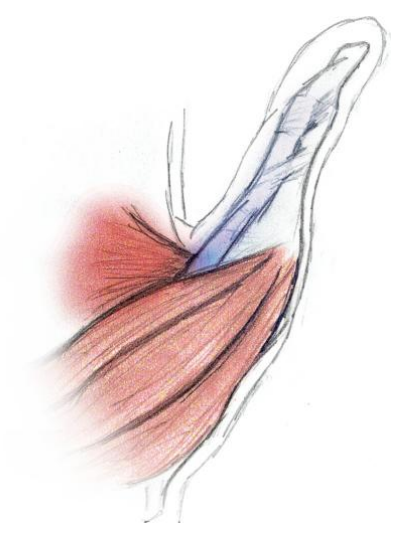

Fig. 4. Thenarian muscles of the thumb and DIP flexor

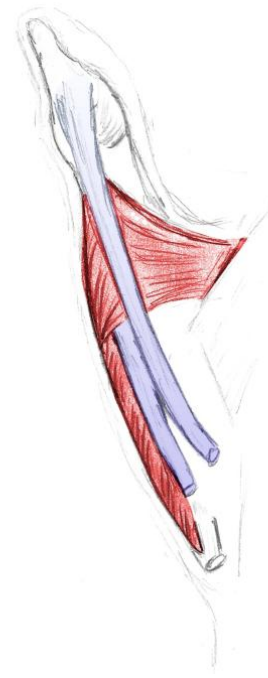

Fig. 5. Extensor apparatus of the thumb

Joints: The joints of the thumb are actuated with muscles and tendons, the fiber hoods are ensuring joint stability. They also provide a certain compliance of the joints, to avoid damages in case of impact. Due to this flexibility and the complex sliding/rolling motion it is difficult to fit properly mechanical joint models. The Distal Inter Phalangeal joint (DIP) is the simplest, considered as a trochlear type [15]. The two other joints, however, have a more complex type. The TM joint is of saddle shape type as reported in [16], and therefore is best approximated by a hyperboloid geometry

\footnotetext{
${ }^{1} \mathrm{~A}$ tendon insertion is the location on the bone where the tendon extremity is attached.
}

joint. This joint must withstand a large pressure (cf. [17]), thus material wear can be an issue in robotic systems. The Metacarpal-Proximal joint has a trochlear type with an important lateral and rotational (twist) degree of freedom. This under-actuated twist motion augments the contact surface during power grasps and improves the fingertip pulp orientation during pinch grasp. However, from a control perspective, a under-actuated motion is more challenging to deal with (and active twist is often expensive to implement) thus a trade-off has to be found between power grasp capabilities and fine manipulation.

The flexion axis of the DIP and MP are not orthogonal to the thumb axis but are a slightly inclined. The exact inclination values are not available, but it results in a tip rotation that improves the tip orientation during opposition motion (similar to the twist of the TM and MP). The inclination rotates the phalanges towards the inside of the palm, preventing contact of the side of the thumb with the object and enabling maximum contact of the fingertip pulp (cf. 6).

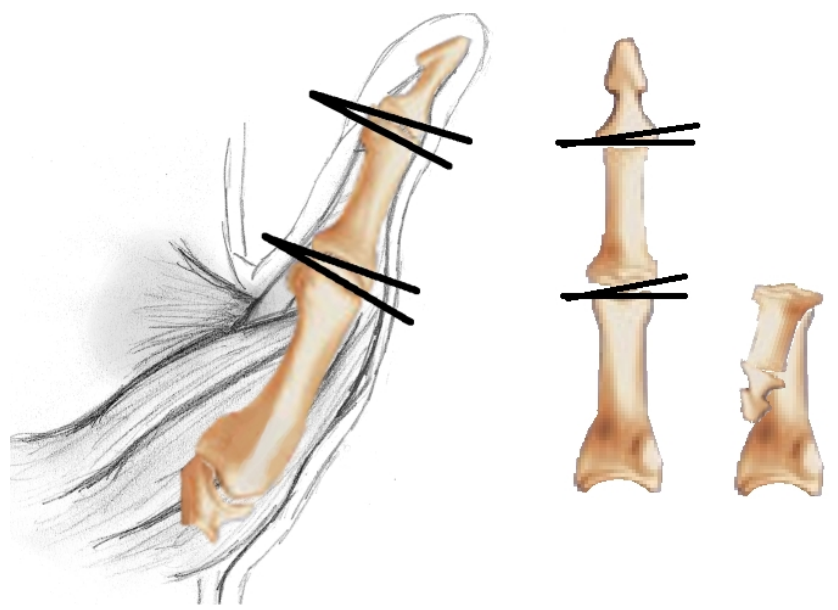

Fig. 6. Rotation (or twist) of the tip orientation due to the joint inclinations

Range of motion: The range of motion (ROM) of the joints varies widely among individuals, but average values can be found in anatomy books [14]. It should be noted that those natural disparities in the ROM are not impairing the manipulation skills. The values are given with respect to the simplified joint models since, as mentioned earlier, the biological joint is difficult to parametrize. The ranges found in [14] (similar to the one reported in [7] ) are reported in table II are given with respect to the approximate reference position described in the figure 7.

Forces: The excursion and tendon forces are more difficult to obtain. Three principal approaches have been identified in the literature but they report a very large variability.

- Kinematic methods based on MRI, X-ray or dissected hands [18].

- Direct tendon forces measurement on cadavers.

- Estimation based on the muscle cross section [19].

- EMG based measurements. 


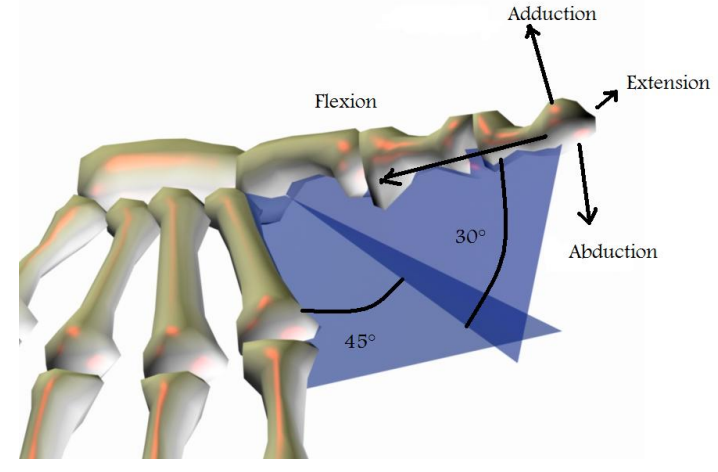

Fig. 7. Approximate reference position of the thumb

TABLE II

AVERAGE ACTIVE RANGE OF MOTION OF THE THUMB JOINTS (EXTRACTED FROM [14])

\begin{tabular}{l|l} 
Digit & $\begin{array}{l}\text { Minimum/Maximum active range } \\
\text { of motion }\left[^{\circ}\right]\end{array}$ \\
\hline MP (Abduction/Adduction) & $-30: 15$ \\
MP (Flexion/Extension) & $-35: 25$ (with 0-8 ${ }^{\circ}$ twist) \\
PIP & $-20: 60$ \\
DIP & $-10: 100$
\end{tabular}

The kinematic methods are extremely sensitive to the location of the rotation center as show by F. Lbath in [17]. A variation of $0.5 \mathrm{~mm}$ in the location of the tendon insertion leads to a variation of $100 \%$ in the estimated forces. In case of direct cadaver measurements the values are known to be biased because of the degradation of tendon sheaths and a forced range of motion. The cross section method (Fick, 1911 and Steindler, 1940), consists in multiplying the muscle cross section by a coefficient $\left(10 \mathrm{~kg} / \mathrm{m}^{2}\right.$ for Fick, $3.65 \mathrm{~kg} / \mathrm{m}^{2}$ for Steindler) to obtain the forces. But it is difficult to apply this to the small muscles of the hand that is why the method is often criticized [15, p.38]. According to Tubiana, the most accurate results have been obtained using EMG stimulation (Freehafer et al., 1979). Those results are similar to Ficks values. The muscles are never working independently and the simplest finger motion is the result of the coordinated action of several muscles. For Kapandji (1963), the movements are the result of the displacement of the equilibrium between muscles groups.

Values for the different tendon forces and tendon excursions can be found in [15, p.38]. However, only their order of magnitude is important for the designer in order to understand the relevant stress that is applied to the joints. Using those values the designer can choose a correct size for the actuators and select the proper material for both tendons and bones. Based on the comparative study of [17], during a $10 N$ load in a pinch grasp configuration, the reaction force on the TM joint are :

- Cooney and Chao (cf. [20]) : 100N

- Giurintano [4]: 180N

- LBath: $70 \mathrm{~N}$
Although the results are widely varying, it appears that the TM joint must withstand large pressure. At the same time, it should maintain a low friction coefficient to improve control performances. Impact forces are naturally larger and a proper mechanical solution is a challenge. In [21, p. 382], it is recalled that the thumb is carrying half of the workload and thus is often subject to wear problems (arthritis).

From the anatomy analysis we can conclude that:

1) The ratio of the bones lengths should satisfy the human like ratios.

2) From a control point of view, the thumb has eight unidirectional actuators and consequently at most 7 degree of freedom of joint motion are controlled.

3) The tendon forces are not simply applied at a point and the tendon routing creates a complex mapping from muscle forces to joint forces.

4) The compression force on the TM is about $100 \mathrm{~N}$.

5) The range of motion are widely varying, average values are reported in Table II.

6) The DIP has a hinge joint topology (1 DOF).

7) The MP has trochlear type of joint (2 DOF).

8) The TM has a saddle joint shape (2 DOF).

\section{SURGERY}

Surgery publications and studies contain rich, but fragmented, information about the thumb characteristics. They focus on function restoration and do not propose a clear overview of the desirable properties of a robotic thumb. The most relevant surgery acts for the functional evaluation are:

- metacarpal proximal joint prosthesis

- ligamentoplasty of the TM [22]

- pollicization

- toe transfer

- bone fusion

- widening of the opening angle (first commissure)

The pollicization or index transfer, is a reconstruction operation of the thumb using the index finger. The index finger is not sectioned but displaced to the metacarpal (TMPIP) of the thumb or the proximal phalanx. Depending on the index finger damages it is also possible to use the middle finger but the aesthetic result is worse. The main objective of the operation is to restore the opposition capability [21]. The final length of the thumb column is kept smaller than the original size. This primarily ensures that the traumatized muscles will have enough force to oppose to the remaining fingers. A too long thumb would result in a poor grasp since the other finger tips are too close [21, p. 385]. The figure 8 shows the results of a pollicization. The functional results are excellent, hand writing is achieved with reasonable accuracy.

The toe transfer operation is similar to the pollicization but using a toe for the transfer. The big toe or the second toe can be transferred using a complete or partial transfer but patients report better results (functionally and aesthetically) with the second toe. The toe phalanxes being shorter than the thumb phalanxes, the total length of the thumb column is not a special concern. 


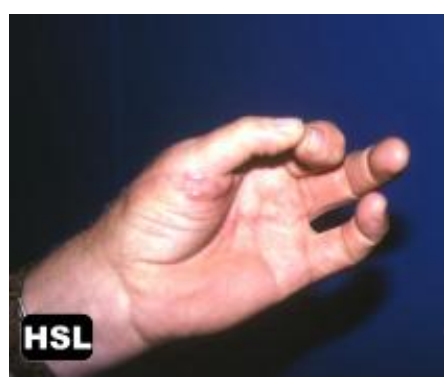

Fig. 8. Hand after a pollicization surgery

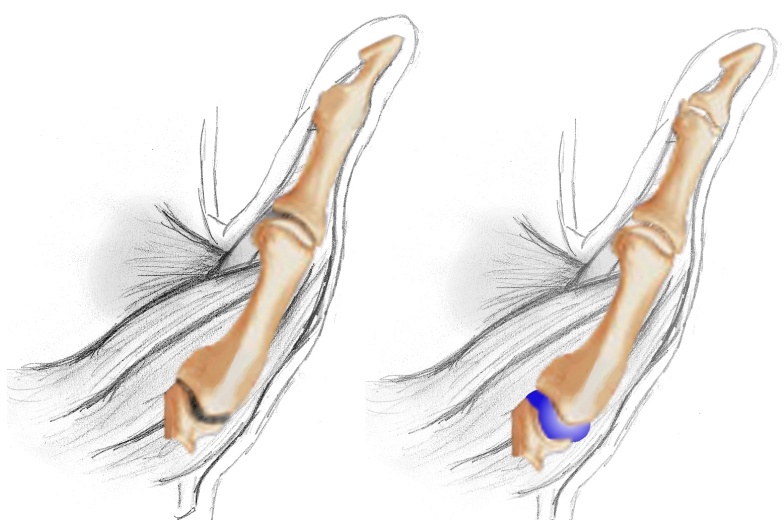

Fig. 9. DIP Joint fusion (left), TM Spherical prosthesis (right)

The bone fusion operation consists in letting the body fuse two bones (or more together), in order to suppress their relative motion (cf. fig. 9, left). This is the privileged approach in case of PIP or DIP arthritis. Indeed, for those joints a prosthetic surgery is complex and leads to a less stable joint than with a fusion. The base bones of the thumb (trapezoid and scaphoid) can be fused or removed to avoid joint pain. The fusion is especially indicated since they have a limited range of motion and the consequences on the manipulation abilities are minimal.

The opposition motion is the main functionality of the thumb and is provided by the TM joint. Therefore, several prosthesis have been developed to replace the trapezoid/metarcarpal joint. They mostly consist either in a simple spherical joint or a ellipsoidal insert( cf. fig. 9, right, for a surgery video, YouTube,Prosthetic Arthroplasty of the Thumb CMC Joint -Dr. Alejandro ). Although the original motion is far more complex [23], prosthesis present very good results. The main difficulty is to position properly the implant such that the risk of subluxation ${ }^{2}$ is minimized. The possibility to conserve the original thumb length as well as a high mobility produces very functional results.

The mobility of the TM is the most important of the thumb, allowing the opposition motion and allowing all configuration between flat hand and opposition grasp (pinch grasp). If the mobility, and stability, of the TM cannot be achieved it will be decided to realize a bone fusion between

\footnotetext{
${ }^{2}$ When the bone head slides out of the usual contact surface, usually due to ligament damages.
}

the metacarpal and the trapezoid. The position is chosen such that a power grasp (of a bottle or a glass) can be achieved. If the other joint are also impaired it is important to offer a sufficient opening angle. In case of low mobility, a shorter thumb is preferable to improve approach angle.

If the first commissure is not large enough (skin band between the thumb and index metacarpals), it can be decided to increase it by a displacement of the insertion point of the intrinsic muscles. In that case the lever of the thenarian muscles and the contact surface between the skin and the objects are reduced. An equilibrium must be found to ensure functionality. A glass or bottle grasp position can be used to guide the choice.

Certainly, the surgery goes beyond the very "mechanical" view proposed in this section. For example, the innervation is essential to provide a proper feedback to the brain and studies involving skin sensitivity impairment demonstrate of this importance. Similarly, blood irrigation is vital to avoid skin or muscle morbidity. However, a correct "mechanical" functionality is the minimum required to build a skilled robotic hand.

From the surgery analysis we can conclude that:

1) The stability of the joints is essential.

2) The opposition motion is provided by the TM joint and is the most important joint.

3) An ellipsoidal implant can be used to restore the $T M$ function.

4) The TM twist motion improves the fingertip pulp orientation during the power grasp.

5) The length of the thumb should be shorter to provide sufficient opposition strength (1 against 3 ).

6) A large opening angle between thumb and index improves reachability but can degrade maximum applicable forces.

7) The tensegrity structure of the thenarian muscles provide high forces as well as stability (prevent subluxation).

\section{Functional Evaluation}

In the context of pre and post surgery evaluation (and also muscle and tendon diseases), tests have been developed to evaluate the performance of the thumb. They range from a very simple force measurement to a complex pick and place task.

Kapandji: The Kapandji test is a very simple test used to control the range of motion of the fingers. The test requires to move the finger to a list of predefined positions (cf. fig 10 from [24]).

Depending on the success or failure of the position, points are awarded (see [25] for an example). The test is especially interesting to test the design because it contains all motion directions (including the opposition motion). With this test the designer ensures that the grasping capabilities are sufficient (at minimum from a kinematic point of view). The scoring scheme can be adapted to reflect the relative importance of each grasp depending on the application. 


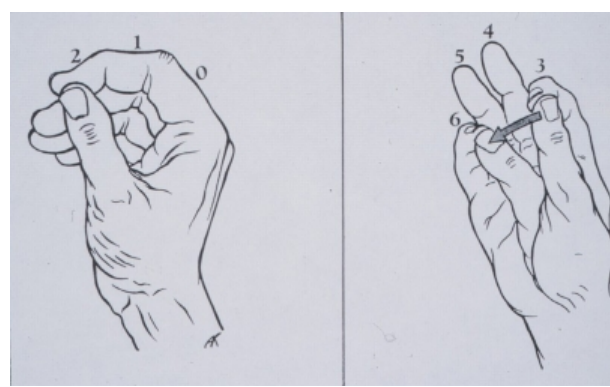

Fig. 10. Kapandji mobility test

Force measurement: In the context of patient follow up, it is useful to keep track of the possible pinch grasp and power grasp forces. The progression or regression of the value (along with a pain evaluation) is a good indicator of the treatment success. The values are often measured using a device similar to those manufactured by Jamar (the tests are often refer to as the Jamar pinch test and Jamar grasp test). The position is not specified during the pinch grasp, so the evaluation of the joint torques and muscles forces is delicate. Nonetheless, it provides a sufficient insight into the force requirement at the finger tip in a half flexed hand configuration. An average force of $130 \mathrm{~N}$ (resp. 110N) are obtained for a human adult male (resp. female).

Take Five: The force, the sensibility and the range of motion are commonly used as indicators for the functionality of the hand. But, for manipulation of small objects, the dexterity is what is important. The question is then to evaluate such a complex criteria. The Take Five test [26] provide an answer to that question. It is, indeed, a very simple test which has been proved to be reliable (the variability in the same person between two trials is small).

The benchmark is composed of a printed pattern and five matches. The goal is to pick up and place the five objects as quickly as possible. The pattern is shown in picture 11. More than just using the total time to pick and place the objects, points are given if the difference in speed between the two hands is small. That way, it is less sensible to the age or the reactivity of the patient. The results presented are especially relevant because they asset the relative importance of each joint.

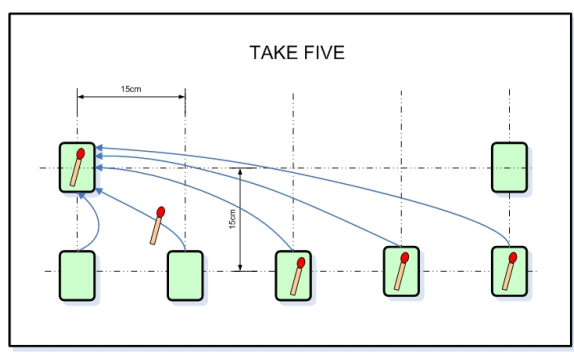

Fig. 11. Test pattern of the take five test

The results of the test depending on the patient condition are reported in the tables III-VII. Patients with Spared Long Digits (SLD) refers to people with at least three completely opposable fingers. From these data some hypothesis can be derived. With the table III, the optimal total active mobility (TAM) is between $20^{\circ}$ and $40^{\circ}$, since larger mobility tends to decrease stability. The table IV leads to a value greater than $30^{\circ}$ for the active range of the PIP whereas the passive mobility of the PIP should not exceed $60^{\circ}$. A very important

TABLE III

SCORE DEPENDing on the Total Active Mobility (TAM)

\begin{tabular}{|c|c|c|}
\hline Number of patients & TAM & Score \\
\hline $3(1 \mathrm{SLD})$ & $0^{\circ}$ & 0.65 \\
\hline 2(1SLD) & $>20^{\circ}$ & 1.5 \\
\hline $4(2 \mathrm{SLD})$ & $>40^{\circ}$ & 2.75 \\
\hline 5(3SLD) & $>60^{\circ}$ & 1.3 \\
\hline 4(2SLD) & $<60^{\circ}$ & 1.5 \\
\hline
\end{tabular}

TABLE IV

SCORE DEPENDING ON THE ACTIVE PROXIMAL-INTER-PHALANGAL (IPP) MOBILITY

\begin{tabular}{|c|c|c|}
\hline Number of patients & Active IPP mobility & Score \\
\hline 7(3SLD) & $0^{\circ}$ & 1.1 \\
\hline 5(2SLD) & $<15^{\circ}$ & 1.8 \\
\hline 5(4SLD) & $<30^{\circ}$ & 1.7 \\
\hline 1(0SLD) & $>30^{\circ}$ & 2 \\
\hline
\end{tabular}

TABLE V

SCORE DEPENDING ON THE PASSIVE PROXIMAL-INTER-PHALANGAL (PIP) MOBILITY

\begin{tabular}{|c|c|c|}
\hline Number of patients & Passive Mobility PIP & Score \\
\hline 4(1SLD) & $0^{\circ}$ & 1.25 \\
\hline 4(3SLD) & $<30^{\circ}$ & 2 \\
\hline 3(2SLD) & $<60^{\circ}$ & 2.2 \\
\hline 7(3SLD) & $<60^{\circ}$ & 1.3 \\
\hline
\end{tabular}

TABLE VI

SCore DePending On the Passive Distal-INTER-Phalangal (DIP) FREEDOM

\begin{tabular}{|c|c|c|}
\hline Number of patients & Active Mobility DIP & Score \\
\hline 13(6SLD) & mobile & 1.6 \\
\hline 5(3SLD) & immobile & 1.57 \\
\hline
\end{tabular}

result for the thumb design is found in the table VI. The mobility or the immobility of the DIP joint has almost no influence on the score. The passive mobility of the DIP must not exceed more than $30^{\circ}$ to provide stability. It should be noted that the base joint (TM) influence is not analysed but its range of motion must allow good Kapandji test results and provide a perfect stability.

From the rehabilitation it can be deduced that:

1) The range of motion evaluated by the Kapandji test are sufficient for good manipulation abilities.

2) The maximum pinch grasp force and the power grasp force are good indicators of the overall force capabilities.

3) The DIP mobility is required only for fine manipulation. 
TABLE VII

Score DePending on the Passive Distal-INTER-Phalangal (DIP) Mobility

\begin{tabular}{|c|c|c|}
\hline Number of patients & Passive Mobility DIP & Score \\
\hline 5(2SLD) & $0^{\circ}$ & 0.6 \\
\hline 7(2SLD) & $<30^{\circ}$ & 2.1 \\
\hline 6(4SLD) & $>30^{\circ}$ & 1.9 \\
\hline
\end{tabular}

4) The TM and PIP active mobility are important to create power grasps, and being able to release them.

5) The passive motion of the joints must be limited in order to preserve stability.

\section{Conceptual Design and application CASE}

The guidelines obtained in the previous sections are sometimes contradictory and, as often, the final design must be careful tradeoff between the desired performance. The desired versability, budget ressources, design time, etc ..., are adding even more terms to the equation. For example, the number of degrees of freedom has a large influence on the ability to perform fine (in hand) manipulation. The underactuated degrees of freedom are providing more anthropomorphism but at the cost of an increased control complexity. The important requirements of the hand of the integrated Hand Arm System are reported in the table VIII.

TABLE VIII

MINIMUM PERFORMANCE REQUIREMENTS FOR THE HAND OF THE HAND ARM SYSTEM (ONLY A SUBSET)

\begin{tabular}{|l|}
\hline Requirement \\
\hline Human size \\
Tendon driven system \\
Good object envelopping (Sec. II, 1) \\
Fingertip manipulation (Sec. IV, 3) \\
Human range of motion (Sec. IV,10) \\
Flat hand configuration \\
Correct magnitude of the thumb forces (Sec. III, 5) \\
Large power grasp : Large Opening angle (Sec. III, 6) \\
Maximum contact surface and proper orientation (Sec. III, 4) \\
Minimal Control complexity
\end{tabular}

Using the previous requirements table, a conceptual design has been created, it is reported in figure 12. The point that should be monitored carefully during the design processed are detailled below.

- Based on the previous sections, the thumb should have at least $3 \mathrm{DOF}$ to allow proper manipulation. The fourth degree of freedom improves the fine manipulation capabilities. Since it is an important requirement, the selected design uses eight tendons (Sect. II, 2) in an antagonistic configuration.

- The TM joint has 2 DOFs and can be implemented as a spherical joint with index or as an ellisoidal joint (Sect. III, 3). It is very important to obtain a stable joint. The MP and DIP joints are hinge joints.

- The twist actuation appears to be too complex with respect to its benefits. To improve the contact orientation during pinch grasps and power grasps, the joint axis of the MP and DIP have been optimized. The resulting hand design presented in fig. 13 is highly compact and robust.

- Inspired by the thenar muscles, a tensegrity structure is considered for the tendons of the TM joint (Sect. III, 7). It results in a larger joint torque and more accurate positioning.

- In order to provide a maximum contact surface between the finger phalanxes and the objets, the ratio of the length of the bones should follow an anthropomorhic scale.

\section{CONCLUSION AND FUTURE WORK}

Although many designs use modular fingers, the thumb should deserve special attention because of its central role in the grasping capabilities of the human. In spite of the great diversity of range of motion, forces and size, hands are capable of similar performances. Therefore, a study to understand the functional aspects of the fingers and the thumb, and summarize it under the form of guidelines has been carried out. The knowledge of the thumb surgery techniques and the precious feedback of thumb surgery patients has been condensed to the most relevant design elements of the thumb. The result of this work has been applied to obtain a conceptual design and applied to the thumb of the Integrated Hand Arm system. Future analysis work will consist in creating software tendon couplings, actuator forces limits and joint range of motion impairment to investigate further their functional influence.

\section{ACKNOWLEDGMENT}

Medical figures have been realised by Jacqueline Porschen.

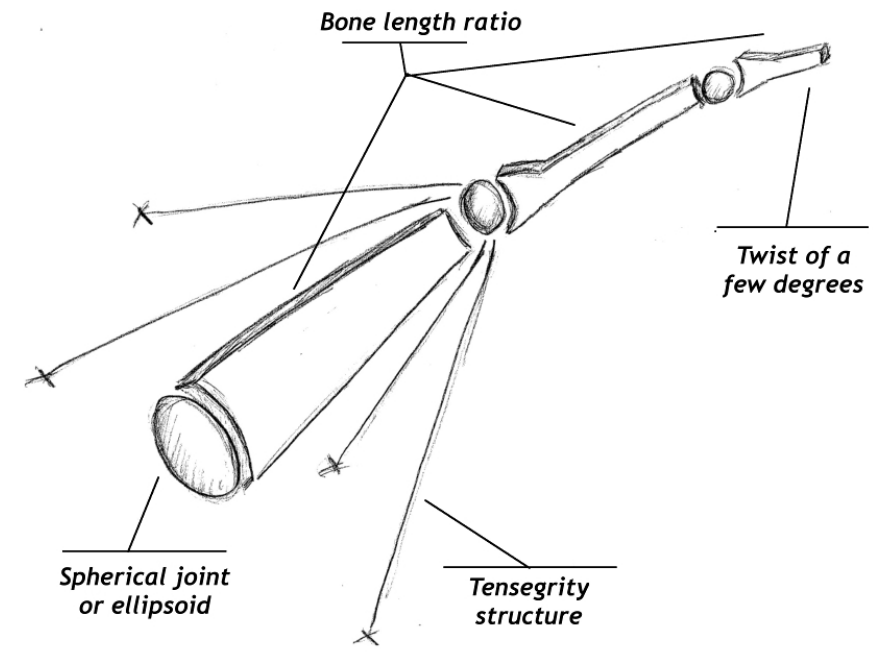

Fig. 12. Conceptual Design Schematic 


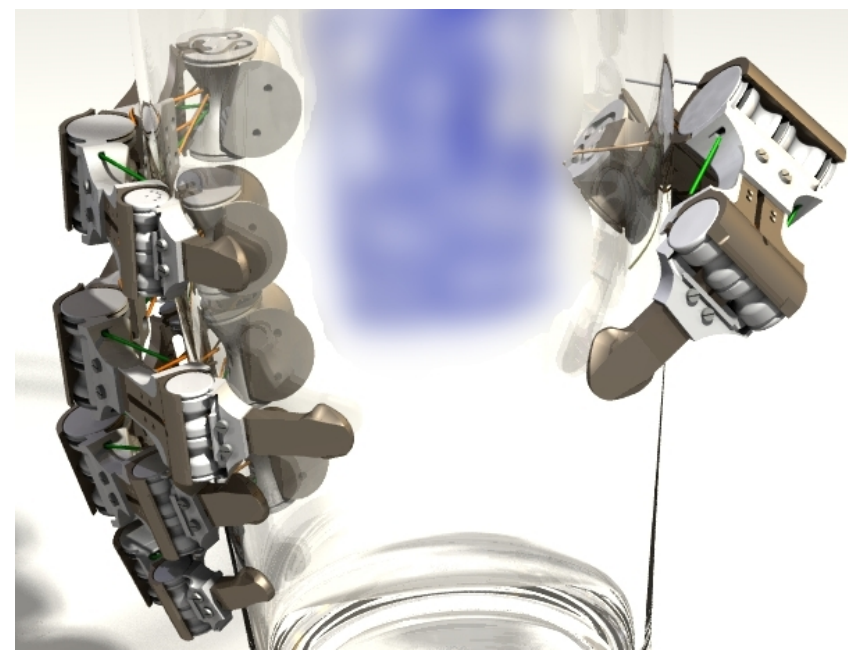

Fig. 13. Joint axes optimized for power grasp contact orientation

This work has been partially funded by the European Commissions Seventh Framework Programme as part of the project The Hand Embodied under grant no. 248587.

\section{REFERENCES}

[1] "Nus group medical insurance scheme (gmis) for nus graduate/nongraduating students," 1 July 2006 - 30 June 2007.

[2] C. Borst, M. Fischer, S. Haidacher, H. Liu, and G. Hirzinger, "DLR hand II: experiments and experiences with an anthropomorphic hand," in ICRA, 2003, pp. 702-707.

[3] Y. Matsuoka, P. Afshar, and M. Oh, "On the design of robotic hands for brain-machine interface." Neurosurgical focus [electronic resource]., vol. 20, no. 5, p. E3, 2006.

[4] D. Guirintano, A. Hollister, W. Buford, D. Thompson, and L. Myers, "A virtual five-link model of the thumb," in Med. Eng. Phys., 1995, vol. 17, pp. 297-303.

[5] V. J.Santos and F. J.Valero-Cuevas, "Reported anatomical variability naturally leads to multimodal distributions of Denavit-Hartenberg parameters for the human thumb," in IEEE Transactions on Biomedical Engineering, February 2006, vol. Vol 53.

[6] J. L. Pearlman and F. J. Valero-Cuevas, "The 3d thumb-tip forces produced by individual tendons do not superimpose linearly," in Proceedings of the 25'. Annual lntemational Conference Of the IEEE EMBS, 1991.

[7] L. Chang and Y. Matsuoka, "A kinematic thumb model for the ACT hand," 2006, pp. 1000-1005.

[8] R. Boulic, S. Rezzonico, and D. Thalmann, "Multi-finger manipulation of virtual objects," in In Proc. of the ACM Symposium on Virtual Reality Software and Technology (VRST '96, 1996, pp. 67-74.

[9] H. Iwata and S. Sugano, "Design of human symbiotic robot twendyone," Proceedings - IEEE International Conference on Robotics and Automation, pp. 580-586, 2009.

[10] P. Tuffield and H. Elias, "The shadow robot mimics human actions," Industrial Robot, vol. 30, no. 1, pp. 56-60, 2003.

[11] H. Kawasaki, T. Komatsu, and K. Uchiyama, "Dexterous anthropomorphic robot hand with distributed tactile sensor: Gifu hand II," IEEE/ASME Transactions on Mechatronics, vol. 7, no. 3, pp. 296303, 2002.

[12] M. Grebenstein and P. van der Smagt, "Antagonism for a highly anthropomorphic hand arm system," Advanced Robotics, no. 22, pp. 39-55, 2008.

[13] R. Hamilton and R. A. Dunsmuir, "Radiographic assessment of the relative lengths of the bones of the fingers of the human hand," Journal of Hand Surgery (British and European Volume), vol. 27, no. 6, pp. 546-548, 2002.

[14] M. Dufour and M. Pillu, Biomécanique fonctionelle. Ed.Masson 2294-01321-2, 2005.

[15] MD. Raoul Tubiana, The Hand. W. B. Saunders Co, 1985.
[16] K. Kuczynski, "The thumb and the saddle," in The hand, 1975, vol. 7 , p. 120.

[17] F. Lbath, C. Rumelhart, and J. Comtet, "Variabilité des forces musculaires et artiulairs de la colonne du pouce. résultats comparés de trois études lors d'un gest ede pince latérale," in Chirurgie de la main, 2001, vol. 20, pp. 11-22.

[18] J. Pearlman, S. Roach, and F. Valero-Cuevas, "The fundamental thumb-tip force vectors produced by the muscles of the thumb," Journal of Orthopaedic Research, vol. 22, no. 2, pp. 306-312, 2004.

[19] R. Linscheid, K.-N. An, and R. Gross, "Quantitative analysis of the intrinsic muscles of the hand," Clinical Anatomy, vol. 4, no. 4, pp. 265-284, 1991.

[20] B. Cooney and E. Chao, "Biomechanical analysis of static forces in the thumb during hand function," The Journal of Bone and Joint Surgery, vol. 59, no. 1, pp. 27-36, 1977.

[21] F. Adrian E.Flatt MD, "Ours thumbs," in BUMC Proceeding, vol. 15 2002, pp. $380-387$.

[22] C. Savornin, C. Boeri, and F. Esling, "Ligamentoplastie trapézométacarpienne : technique personnelle," in Chir. Main, 2001, no. 20, pp. 71-74.

[23] A. Kapandji, "Biomécanique des articulations trapézométacarpienne et scaphotrapézienne," in Monographies du GEM, La Rhizarthrose, E. S. Française, Ed., 198x, no. 16, pp. pp. 33-48.

[24] _ "Cotation clinique de l'opposition et de la contre-opposition du pouce," in Annales de Chirurgie de la Main, 1986, vol. 5, no. 1, pp. $68-73$.

[25] X. D. Soras, D. Guinard, F. Moutet, and P. Gerard, "Fiche de cotation fonctionelle," in Ann. Hand Surg, 1994, vol. vol.13, no. no5, pp. 297307.

[26] R.Selinger, V.Mitz, J.P.Lemerle, and R.Vilain, "Un nouveau test d'évaluation fonctionelle de la main et son apport dans l'étude des transferts d'orteil : le test des 5 allumettes dit : Take five," in Ann. Chir. Main, 1991, vol. n2, no. 10, pp. 124-137. 